\title{
Cerebral palsy risk factors: international experience
}

\author{
Raushan Issayeva ${ }^{1}$, Aigerim Aliakparova ${ }^{1}$, Symbat Abzaliyeva ${ }^{1 *}$, Gulzhan Kassenova ${ }^{1}$, \\ Gulnara Tashenova ${ }^{1}$ \\ ${ }^{1}$ Al-Farabi Kazakh National University, al-Farabi Ave. 71, 050040 Almaty, Republic of Kazakhstan
}

\begin{abstract}
This review article aims to outline several risk factors for the Cerebral Palsy (CP) development worldwide. CP is the most prevalent disabling condition in children that imposes a significant socio-economical responsibility on the system of the health care. Despite a solid body of extant research, the exact etiology of $\mathrm{CP}$ remains unknown. There are several risk factors that may be triggering $\mathrm{CP}$ development at pre-, intra- and postnatal periods, particularly, gestational age, birth weight, mother's health, placental abnormalities, thrombophillia, asphyxia, brain ischemia and multiple pregnancies. According to extant literature, the majority of $\mathrm{CP}$ cases develop within antenatal period in high-income countries. Contrastingly, in developing countries, there is a slightly higher proportion of a postnatally acquired CP cases linked to post-infectious brain damage following meningitis, septicaemia, as well as other conditions, such as malaria. However, these studies were of a small size and not case-controlled or population-based, which significantly curtails the results and underestimating the real picture. With very small number of survivors of early preterm, common risk factors identified to be the maternal rhesus allergenic immunization and birth asphyxia, or hereditary diseases, such as dehydrogenase of glucose-6-phosphate (G6PD) deficiency and encephalopathy of subsequent bilirubin. According to standardized data from international surveillance programs, important risk factors are strongly associated with $\mathrm{CP}$ development in most countries.
\end{abstract}

\section{Introduction}

Cerebral palsy (CP) is a non-progressive disorder of action arising from neural injury during the perinatal period, associated with a wide range of common corresponding morbidities. Interventions in early childhood are of great benefit, when brain plasticity is at its greatest, so that developmental trajectories can be changed with maximal benefit into adulthood. Collaborators within Surveillance of Cerebral Palsy in Europe (SCPE) have created a special database, that is a very useful tool for monitoring CP epidemiology and providing a framework for etiological and health services research. The database contains information on 3.3 million live birth cases out of approximately 17 million people

\footnotetext{
*Corresponding author: Symbat.Abzalieva@kaznu.kz
} 
suffering from $\mathrm{CP}$ worldwide [1]. On the basis of massive research net, researchers have successfully lowered of the cerebral palsy diagnosis age to 19 months. However, in majority clinical settings, the age for CP diagnosis is two years on average or beyond. Late $\mathrm{CP}$ diagnosis is associated with parental unsatisfaction, and higher rates of mental health conditions, including depression. The average age for diagnosis is about 18 months; latest evidence suggests that cerebral palsy can be detected as early as three to four months using tests such as Prechtl's Qualitative Assessment of General Movements and magnetic resonance imaging [2]. As of today, $\mathrm{CP}$ remains a clinical description, but registries and surveillance programs, such as those in Australia, United Kingdom, and Europe, highlight five main elements reflecting the essential features of cerebral palsy, provided in definitions, and proposed by the SCPE: (i) it is an 'umbrella term'; (ii) it is permanent but not invariable; (iii) it involves restriction disorder of movement, posture, motor function, or a combination; (iv) it is attributed to a non-progressive interference, lesion, or abnormality; and (v) the interference, lesion, or abnormality arising in the developing or immature brain $[3,4]$. The exact etiology of this pathology remains unknown, although some research suggests that the mother's health, environmental, genetic and different ante, peri and post-natal factors could underlie the development of CP. Therefore, this review will outline several one of the most prevalent risk factors for CP development in high and low-income countries.

\section{Methods of research}

Achieving the goal and solving the tasks set in the study was carried out using methods of logical and comparative analysis, systematization, statistical analysis, evaluation and theoretical generalization.

\section{Results and Discussion}

\subsection{Cerebral Palsy Risk Factors in low-income poor-resources countries}

It should be underlined that $\mathrm{CP}$ causes and risk factors may differ in low-and middle-income and in developed countries. Some authors claim that perinatal (e.g. asphyxia, sepsis) and postnatal causes such as head trauma, meningitis and hyperbilirubinemia devote a large proportion of disease in developing countries. Similarly, in the high-income rich-resource countries, CP is more often related to survival rates of prematurity and prematurity itself [5, 6]. In addition, most reports from low-income countries are mainly based on either hospitals or clinics and recruit an insufficient number or narrower age-range of patients [7]. Recent study from India showed that $57.4 \%$ of 1212 cases were attributed to CNS infections such as encephalitis and meningitis, 30\% to bilirubin-encephalopathy (usually G6PD deficiency or secondary to Rh-incompatibility), $7.4 \%$ to intracranial bleeds or late hemorrhagic disease of newborn; meanwhile the rest were secondary to head injury and hypoglycemia. Other factors were also found to contribute, especially to prematurity $(13.2 \%-24.3 \%$, p-value 0.0001$)$, birth asphyxia $(45.3 \%-51.98 \%$, p-value 0.001$)$, neonatal jaundice $(21.6 \%-35.14 \%$, p-value $0.0001)$ and low birth weight (20.4\%-37.87\%, p-value 0.0001)[5].

However, recent research from Russia also demonstrated that the most significant antenatal factors include low birth weight (LBW), abortions in anamnesis and severe gestosis [8]. In contrast to other more developed countries data, no association was identified for infections and subsequent CP development (OR 3.464, CI = 2.144-5.596), but there was a strong association between premature birth and prolonged anhydrous period (OR 8.043 (CI 
- 3.348-9.252 and OR 11.505 (CI - 5.218-13.652), respectively [8]. For post-natal risk factors, no strong association was identified for septic conditions (OR, 1.473 (CI $=1.221-2.362$ ), hemolytic disease of newborns (OR $1.637(\mathrm{CI}=1.238-2.879)$ and traumatic lesions of the spinal cord, peripheral nervous system and brain OR $1.413(\mathrm{CI}=1.118-2.009)$ [8]. A recent study from Pakistan demonstrated that the important risk factors in the country included preterm delivery, consanguinity and infections during pregnancy [9]. In Palestine, three risk factors of $\mathrm{CP}$, namely, consanguineous marriage, delivery at private maternity homes lacking emergency facilities, and no prenatal doctor visits were identified as the most significant risk factors [10].

Moreover, as a recent systematic search of studies published between 1990 and 2009 in databases such as on Ovid, Cinahl, PubMed, SCOPUS, the Cochrane database, and data from international disability organizations and hospital-based studies showed that $\mathrm{CP}$ rates in population-based settings in developing countries such as China, India, countries of South America and East Asia varied from 2-2.8/1000 births, which is analogous western settings [6].

Nonetheless, CP studies performed hospital-based showed heightened rates of spastic quadriplegia in contrast to hemiplegia or diplegia, and possibly increased rates of asphyxia, meningitis and jaundice, and rates of low birth weight and in CP populations prematurity [7]. With low survivors of early preterm births in thouse countries, typical risk factors identified were maternal rhesus alloimmunisation and birth asphyxia, or inherited disorders, such as subsequent bilirubin encephalopathy and glucose-6-phosphate dehydrogenase deficiency. In addition, higher proportions of children were associated with postnatal acquired CP linked to post-infectious brain damage following septicaemia, meningitis and coresponding conditions, such as malaria. However, these studies were of a small size neither case-controlled nor population-based, which limits the results significantly, thus underestimating the real picture [5].

\subsection{CP in developed and high-income countries}

In contrast to the above, according to a recent retrospective study of Japanese National Registry data, the most important CP risk factors in Japan were placental abnormalities (31\%), umbilical cord abnormalities (15\%) as well as maternal and neonatal complications (10\% and 1\%, respectively) [11]. According to a new multivariate regression model, obstetric variables associated with $\mathrm{CP}$ were acute delivery due to the non-reassuring fetal status (relative risk [RR]: 37.182, 95\% confidence interval [CI]: 20.028-69.032), uterine rupture (RR: 24.770, 95\% CI: 6.006-102.160), placental abruption (RR: 20.891, 95\% CI: $11.817-$ 36.934), and preterm labor (RR: 3.153, 95\% CI: 2.024-4.911). On the other hand, head presentation (RR: 0.199, 95\% CI: 0.088-0.450) and elective cesarean section (RR: 0.236, 95\% CI: 0.067-0.828) were identified as protective factors [11].

According to the previous research data, brain injury in approximately $6 \%$ of individuals with $\mathrm{CP}$ occurred within the postnatal period, particularly, during or more than 28 days after the birth. For the remaining $94 \%$, brain injury occurred during pregnancy, birth or the first 28 days of life [12]. Preterm delivery represents one of the principal risk factors for CP and associated neurosensory disabilities, which is evidenced in the fact that over $40 \%$ of individuals with cerebral palsy are born preterm [13].

For instance, preterm delivery and low birth weight were found in $59.51 \%$ and $60.28 \%$ of subjects with CP in South Korea [14]. Postnatally acquired CP accounted for $15.3 \%$ where hypoxic ischemic encephalopathy was found to be the most common event; moreover, $13.20 \%$ subjects were born from multiple births [14]. 


\subsection{Common risk factors for CP development for high and low-income countries}

Nonetheless, it should be underlined that the exact $\mathrm{CP}$ etiology remains unclear and continues to be a subject of research. According to systematic review that used accurate methodology, searching common bibliographic databases (such as CINAHL, MEDLINE, Science Direct and Cochrane library) with a proper stated search strategy, including work published from 1985 to 2006 while exept those with participants born prior to 1985 that analyzed 49 studies worldwide (with varying ages of ascertainment), the global pooled CP prevalence is 2.11 per 1000 live births (95\% CI 1.98 to 2.25). Moreover, a cumulative meta-analysis demonstrated stability over the past 10 years [15]. The prevalence of CP stratified by age of gestational reported the major pooled prevalence to be among children with mass $1000 \mathrm{~g}$ to $1499 \mathrm{~g}$ at birth (95\% CI 53.06-66.01; 59.18 per 1000 live births), despite no significant difference found among meta-regression pairwise children with body mass less than $1000 \mathrm{~g}$. The predominance of CP expressed by age of gestation was more prevailing among children that were born before 28 gestation weeks (111.80 upon 1000 live births; 95\% CI 69.53-179.78; p<0.0327). Whithin the aforementioned meta-analysis, the pervasiveness was found to be the highest in children with body mass 1000 to $1499 \mathrm{~g}$ at birth (59.18 per 1000 live births), as well as in children born before 28 gestation weeks (111.80 per 1000 live births [15].

According to reports, $\mathrm{CP}$ prevalence estimates tended to be in a similar range, in low-and middle-income countries or higher; however, comparing rates is a challenging endeavor as only small number of these countries use registries or surveillance programs $[16,17]$. According to the Australian Cerebral Palsy Register, with the inclusion of information from 1993 to 2006, an overall CP prevalence of 2.1 per 1,000 live births was reported with high prevalence in multiples ( 7 per 1,000 live births) and in extremely low birthweight neonates (e.g., for birthweight $<1,000 \mathrm{~g}$, the prevalence was 50 per 1,000 live births) $[18,19]$. Nevertheless, more than half of all individuals with CP were born at term [19]. Many studies on antenatal, intrapartum and neonatal risk factors for $\mathrm{CP}$ have identified a great number of commonly reported risk factors, which include: i) factors prior to conception, e.g. low or progressive age of mothers, high unity, absence of parity, a short or long intervals between pregnancy, cases of deadbirth, number of miscarriages, death of neonates or preterm birth, CP history in the family and other genetic susceptibility, low socioeconomic status, as well as pre-existing maternal conditions (such as epilepsy or intellectual disability); ii) factors in early pregnancy, e.g. male sex, number of gestation, birth defects and infections (such as rubella, toxoplasmosis parasite, cytomegalovirus, Zika virus, herpes simplex virus, [20,21]; iii) factors during pregnancy, e.g. maternal disease including thyroid disorders, pregnancy complications (such as pre-eclampsia, placenta praevia and placental abruption), intrauterine infection or inflammation and chorioamnionitis, intrauterine growth restriction, smoking [22] and other precursors to preterm birth; and iv) factors both among the birth time and neonatal period, e.g. acute intrapartum hypoxic events and neonatal encephalopathy, neonatal brain injury (such as intraventricular haemorrhage, periventricular leukomalacia and hydrocephalus), strokes or seizures, cardiovascular disorders (such as patent ductus arteriosus and hypotension), respiratory disorders and associated prolonged ventilation (including respiratory distress syndrome or bronchopulmonary dysplasia), infection (such as sepsis and necrotizing enterocolitis), metabolic or endocrine disorders (including hypoglycemia and hypothyroidism), neonatal jaundice, in addition to inborn errors of metabolism, particular syndromes or chromosomal abnormalities [23].

Research has revealed some contentiousness on the role of birth asphyxia being either a relatively rare cause [24] or being a principal risk factor for CP [25]. Common risk factors in the post-neonatal period involve: infection (such as meningitis/encephalitis or severe 
infection and subsequent severe dehydration), head injury (such as those induced from traffic accidents, other traumatic injury, or non-accidental injury), vascular episodes (such as post cardiac or brain surgery), as well as other events (such as near drowning or near sudden infant death) [4]. Nonetheless, according to a recent systematic review by McIntyre and colleagues, 10 out of 38 risk factors were consistently associated with a statistically significant $(p<0.05)$ risk of CP in infants. These include abnormalities of the placental, defects received during the birth, meconium aspiration, small weight upon birth, birth asphyxia, instrumental / emergency Caesarean delivery, birth asphyxia, respiratory distress syndrome, neonatal seizures, neonatal infection and hypoglycemia [25].

Notably, the term 'birth asphyxia' is non-specific, and it may be pertinent to point out that infants thought to be suffocated at birth could be found to have other diagnoses such as non-asphyxial encephalopathy or sepsis. In this regard, only a small number of papers showed combinations of risk factors and their interactions, but some combinations of several risk factors taking place simultaneously may not only promote but also exaggerate $\mathrm{CP}$ development; therefore, further research within a causal pathway framework should investigate factors that may act synergistically.

Table 1 summarizes common ante-, intra- and postnatal risk factors for the $\mathrm{CP}$ development.

Table 1. Main common risk factors in ante, intra- and post-natal periods for $\mathrm{CP}$ development.

\begin{tabular}{|c|c|c|}
\hline $\begin{array}{l}\text { CP risk Factors } \\
\text { Antenatal period }\end{array}$ & & References \\
\hline \multirow[t]{15}{*}{ Antenatal period } & $\begin{array}{l}\text { Very low birth weight (OR 4.003, (CI) 3.545-4.520, } \\
\text { OR -39.502 (CI = 28.076-55.579) }\end{array}$ & {$[15,16,18,13,19,31]$} \\
\hline & Threat of abortion OR $4.712(\mathrm{CI}=3.705-5.992)$ & {$[1,7,8]$} \\
\hline & $\begin{array}{l}\text { Abortions in the anamnesis (including spontaneous) } \\
\text { OR равняется } 8.143(\mathrm{CI}=5.975-11.098),\end{array}$ & {$[16,18]$} \\
\hline & $\begin{array}{l}\text { Severe gestosis (hypertension, edema, nephropathy) } \\
\text { (OR } 10.776 \text { (при CI = 7.286-15.398) }\end{array}$ & {$[25]$} \\
\hline & $\begin{array}{l}\text { Cold or flu during the pregnancy (OR равняется } \\
3.464(\mathrm{CI}=2.144-5.596))\end{array}$ & {$[8,38]$} \\
\hline & $\begin{array}{l}\text { Umbilical cord initiation with intrauterine hypoxia } \\
\text { development (OR } 1.223(\mathrm{CI}=0.869-1.669))\end{array}$ & [11] \\
\hline & Multiple pregnancy OR $3.464(\mathrm{CI}=2.144-5.596)$, & {$[37,38]$} \\
\hline & $\begin{array}{l}\text { Intrauterine infections (TORCH infections), genital } \\
\text { tract infections OR } 1.381(\mathrm{CI}=0.944-2.028)\end{array}$ & [5] \\
\hline & $\begin{array}{l}\text { Polyhydramnios and aridity OR } 1.586(\mathrm{CI}=1.011- \\
2.488) \text {, }\end{array}$ & [8] \\
\hline & $\begin{array}{l}\text { Intrauterine hypoxia-ischaemia of the brain, } \\
\text { intraventricular and intracerebral hemorrhages, } \\
\text { cysts of the brain (OR } 2.801 \text { (CI - } 1.714-4.576)\end{array}$ & {$[26,27,30]$} \\
\hline & $\begin{array}{l}\text { Inadequate fetal position in uterus (OR } 2.629 \text { (CI - } \\
0.932-7.411) \text {,) }\end{array}$ & [8] \\
\hline & 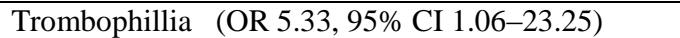 & {$[33,38]$} \\
\hline & $\begin{array}{l}\text { Taking terratogenic medications during pregnancy ( } \\
\text { OR } 3.707, \mathrm{CI}=1.030-13.342)\end{array}$ & [36] \\
\hline & $\begin{array}{l}\text { Placental abnormalities ( RR: 20.891, 95\% CI: } \\
\text { 11.817-36.934, }\end{array}$ & {$[11]$} \\
\hline & 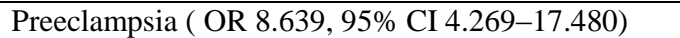 & [34] \\
\hline Intranatal period & $\begin{array}{l}\text { Premature birth (OR } 8.043 \text { ( CI - 3.348-9.252), } \\
\text { uterine rupture (RR: } 24.770,95 \% \text { CI: 6.006- } \\
\text { 102.160), }\end{array}$ & [11] \\
\hline
\end{tabular}




\begin{tabular}{|l|l|l|}
\hline \multirow{2}{*}{$\begin{array}{l}\text { Prolonged anhydrous period (OR 11.505 (CI - 5.218- } \\
13.652)\end{array}$} & {$[7,8]$} \\
\cline { 2 - 3 } & Asphyxia & {$[24]$} \\
\hline Postnatal period & $\begin{array}{l}\text { Traumatic lesion of the spinal cord, brain and } \\
\text { peripheral nervous system OR 1.413 ( CI = 1.118- } \\
2.009),\end{array}$ & {$[18]$} \\
\cline { 2 - 3 } & $\begin{array}{l}\text { Influence of indirect bilirubin on subcortical } \\
\text { structures (OR 4.969 }(\mathrm{CI}=2.538-6.469)\end{array}$ & {$[5,6,7]$} \\
\cline { 2 - 3 } & Hemolytic disease of newborns & {$[6,7]$} \\
\cline { 2 - 3 } & Septic conditions OR 1.473 (CI =1.221-2.362) & {$[18]$} \\
\hline
\end{tabular}

\subsection{Hypoxia-ischaemia}

Brain injury is attributed to multiple causes such as hypoxia-ischemia, hemorrhage, infection, maldevelopment, and metabolic disorders [26]. In particular, brain hypoxia (oxygen deficiency) and ischemia (insufficient blood supply) can lead to severe neuropathology in preterm and born at term infants, especially with the damage of the white matter of the cerebral dominating in the infants born pretermed, and injury in neouranal in the stage of infants [26]. Injury caused to the developing brain believed to be associated with long-term residuals such as $\mathrm{CP}$, problems with hearing, sight, speech, and behavioral disorders, seizures as well as intellectual disabilities [27].

The degree of prematureness is link with the cerebral white matter vulnerability, and is predictive of a heightened risk of the injury of the white matter (such as periventricular leukomalacia), and IV hemorrhage, which are known to be established risk factors for the development of CP [28]. Very low birth weight (VLBW) children are predisposed to the risk of both structural brain abnormalities and severe neurocognitive deficits [15, 28].

\subsection{Pre-term birth}

Preterm birth is accepted as a main risk factor for $\mathrm{CP}$, reflected in the fact that over $40 \%$ of individuals with $\mathrm{CP}$ are born preterm; nonetheless, up to $60 \%$ of individuals with $\mathrm{CP}$ were born at term [8]. New evidence suggests that around $70 \%$ to $80 \%$ of the cases of CP depends on antenatal factors, with birth asphyxia playing aapproximately lower role [27]. Moreover, a recent population-based study showed that there is an increased risk of CP associated with racial and socio-economic disadvantages; therefore, the risk of spastic $\mathrm{CP}$ was more than $50 \%$ higher for Black versus Caucasian race representatives (OR 1.52, 95\% CI 1.33-1.73), whereas there was a lower risk of mothers in high education (OR 1.35, 95\% CI 1.18-1.55) [29]. The birth prevalence of CP is far higher in preterm than in term infants, increases with decreasing gestational age at delivery, and can reach up to $15 \%$ among preterm neonates born between 24 and 27 weeks of gestation. Indeed, in 1,000 live births, the prevalence of CP among neonates born prior to 28 weeks of gestation is 82 , which reduces to 1.4 at 36 weeks of gestation. In addition, with preterm delivery being an established risk factor for $\mathrm{CP}$, a recent study suggests that post-term pregnancy such as 42 weeks or even later can also be associated with CP development. Furthermore, the impact of fetal growth restriction on the prevalence of $\mathrm{CP}$ is also well established.

The prevalence of $\mathrm{CP}$ among neonates weighing $<1,500 \mathrm{~g}$ is $59.2 / 1,000$ live births, as compared to $1.33 / 1,000$ live births among those weighing $>2,500 \mathrm{~g}$ [15]. This association is independent of gestational age at delivery and extenuated by the presence of congenital anomalies, especially of the central nervous system [30]. In an Australian population-based study of singleton pregnancies delivered $\geq 35$ weeks of gestation, the authors examined 494 children with CP. Growth restriction was prevalent in $16.5 \%$ of the neonates with $\mathrm{CP}$, 
whereas the odds of these children to be growth restricted in comparison to those without was 3.5 (95\% CI 2.2-5.5). Correspondingly, a population-based study in Denmark reported a CP prevalence of 1.95/1,000 live births. CP was also associated with an increased head/abdominal circumference ratio and cephalic index regardless of gestational age [30]. For this reason, the intrauterine processes associated with fetal growth restriction also leads to neuronal damage and subsequent $\mathrm{CP}$ development.

\subsection{Gestational age and Birthweight}

Furthermore, the development of registers for CP showed that its prevalence is backwards depends upon weight during the birth and gestational age, with the dominance pasturing from 90 cases per 1000 neonatal survivors weighing less than $1000 \mathrm{~g}$ to $1-5$ cases per 1000 for those whose birthweight was at least $2500 \mathrm{~g}$ [31]. The upper age limit used for the definition of postneonatal cerebral palsy is arbitrary, but it is considered to be about 5 years in the majority of studies. Notably, about $10 \%$ of overall CP cases are classified as postneonatal, that are predominantly matured to CNS infections such as different head traumas and meningoencephalitis [31].

\subsection{Birth asphyxia and developmental abnormalities}

Birth slumber resulting in neonatal encephalopathy and coresponding CP development is often considered to be attributed to physical damage occurring during the labor; however, it only accounts for $2-10 \%$ cases of CP [32]. Research showed that a majority of these pregnancies complicated with "birth asphyxia" already had abnormal placental pathologies leading to fetal vascular disruptions, which resulted in severe neurologic disability appearing later on in life. A study from Scotland examined the post-mortems brain tissue of infants, who died within a couple days (after the birth) from hypoxic ischemic encephalopathy.

Fifty percent of infants examined developed pathological alterations in the brain tissues such as cyst formation and severe calcification. Therefore, it is possible that some fetuses may already have had brain damage before entering the labor. Moreover, around $12 \%$ of CP kids register by SCPE were identified to developed malformation of the brain. Some of the malformations may be accomplished (e.g. ischemic lesion in first or second trimester affecting cortical migration), although others could have a genetic origin. Moreover, factor $\mathrm{V}$ Leiden, Protein $\mathrm{C}$ or $\mathrm{S}$ deficiency are also risk factors that may affect subsequent pregnancies [33].

\subsection{Placenta-Mediated Pregnancy Complications}

According to the data, no single catastrophic placental event with the exception of retroplacental haemorrhage is strongly associated with CP development [4]. However, some research has demonstrated that obstetric variables linked to $\mathrm{CP}$ were acute delivery due to the non-reassuring fetal status (relative risk [RR]: 37.182, 95\% confidence interval [CI]: 20.028-69.032), uterine rupture (RR: 24.770, 95\% CI: 6.006-102.160), placental abruption (RR: 20.891, 95\% CI: 11.817-36.934), [11]. Meanwhile several other broad placental pathologies such as vascular development, acquired inflammatory lesions, and acquired degenerative lesion, which are usually thrombotic in nature, may also trigger $\mathrm{CP}$ development, although these risk factors must be investigated by further research [8]. Nonetheless, maternal genitourinary tract infections caused during pregnancy significantly increases the risk of CP development [11]. 


\subsection{Multiple Gestations, Twins}

Development and practical application of in-vitro fertilization (IVF) as well as assisted reproductive techniques showed that $\mathrm{CP}$ development can also be attributed to multiple gestations. According to embolic and ischemic theories, the transference of thromboplastin or thromboembolin from the dead fetus to the living co-twin may be associated with cerebral damage in the living fetus [35]. However these theories do not seem to help to explain CP development in dichorionic or singletons multiple pregnancies, obstetric ultrasound has revealed that one or more embryos from a multiple conception can be lost early in pregnancy as a vanishing twin or triplet; therefore, this twin could be the cause of CP and other disorders in several singleton births [36]. Indeed, among twin gestations, an affected twin with CP exacerbates the risk that the other fetus will develop CP by 15 times [36].

Moreover, even if in utero death of one twin occurs early in gestation, it is associated with markedly increased risk for $\mathrm{CP}$ in the surviving co-twin [37]. In this regard, an Australian study showed that the prevalence of CP in singletons, twins, and triplets were 1.6, 7.3 , and 28 per 1,000 births, respectively [38]. The authors also reported that in the case of co-twin death, the risk for CP increases by a factor of 8 from 12/1,000 to 96/1,000 [38]. Therefore, a growing body of literature shows that transfusion problems between fetuses might also provide a unified pathogenic mechanism in some cases of CP development both in pre-term and born at term infants.

\subsection{Perinatal Fetal Stroke and thrombophilia}

A study by Gunt and colleagues showed that $39 \%$ of the 100 full-term neonates diagnosed with neonatal arterial ischemic stroke were diagnosed with CP at the age of two [39]. In a report from California, USA encompassing 36 children with arterial ischemic stroke, about $58 \%$ of them developed CP further in life. At present, no clear mechanisms leading to perinatal strokes are understood, though preeclampsia and placental vascular disorders such as thrombophilia are being proposed as risk factors. However, the association between CP and thrombophilia is still under investigation. An Australian population-based case-control study did not show any association between a single thrombophilic mutation and CP [33]. In particular, among term and pre-term neonates with the methylenetetrahydrofolate reductase (MTHFR) C677T mutation, those who were homozygous and born between 32 and 36 weeks of gestation had an odd ratio (OR) of 2.55 (95\% CI 1.12-5.74), while the heterozygous ones had an OR of 1.91 (95\% CI 1.01-3.66) to develop any type of CP [33]. This MTHFR mutation at the homozygous state, among preterm neonates born $<32$ weeks of gestation, was also associated with increased risk for diplegia (OR 2.76, 95\% CI 1.21-6.12), whereas the heterozygous was associated with a mildly increased risk for diplegia throughout gestation (OR 1.58, 95\%, CI 1.02-2.45). A different mutation, MTHFR A1298C at the heterozygote state, was associated with risk reduction for diplegia in neonates born at 32-36 weeks of gestation (OR $0.16,95 \%$ CI $0.02-0.70)$. Among early preterm neonates ( $<32$ weeks of gestation), factor V Leiden (FVL), homozygosity could be related to an increase in the risk of developing quadriplegia (OR 9.12, 95\% CI 0.86-53.71). Heterozygous Prothrombin Gene Mutation (PGM) and homozygous MTHFR C677T combined were related to quadriplegia at all gestational ages (OR 5.33, 95\% CI 1.06-23.25) [40].

On the other hand, an Israeli retrospective case-control study did not find any increased prevalence of thrombophilic mutations among children with CP [41]. The association between perinatal stroke and subsequent $\mathrm{CP}$ development is suggestive of the fact that there might be a strong genetic background in some cases, leading to $\mathrm{CP}$ development; however, much more systematic study should be performed in the field of CP genetics in order to draw better informed conclusions. 
Presence of the infections, such as Trichomonas, Chlamydia and urinary tract infections collectively increase the risk of CP development [24]. Moreover, a recent population-based cohort study demonstrated that early-onset preeclampsia can be studied as a seperate risk factor for CP (OR 8.639, 95\% CI 4.269-17.480) afterreadjustment for gestational age at delivery and the fetal growth restriction [34].

\subsection{Encephalopathy of Prematurity (EOP)}

Encephalopathy of prematurity is a leading risk factor for developing CP from 20-60\% of individuals with EOP who eventually develop CP [36]. EOP is a complicated amalgam of various disturbances involving both gray and white matter injuries in different brain regions caused by intrauterine systemic infection and / or hypoxia-ischemia [42]. White and gray matter lesions are the most prevalent type of injury observable in the magnetic resonance images of children with $\mathrm{CP}$, with a correlation of quantitative damage of gray and white matter lesions with the severity of motor and cognitive deficits in children with CP [42]. White matter injury, or so-called, periventricular malacia, is a well-established prognostic factor for CP development [30].

Severe brain lesions might be related to excitotoxicity microglial activation, reactive gliosis, the express of free radicals, as well as subsequent hypomyelination that can be detected by ultrasound and MRI. On the other hand, the prevalence of EOP is dismissed, and is could be seen in only $5 \%$ of survivors with very-low-birthweight; nonetheless, diffuse PVL is currently the most frequent type of lesion observed in $90 \%$ of very-low-birthweight survivors who eventually develop CP [43].

\section{Conclusion}

Cerebral palsy is a neurologic motor disorder that can be attributed to several underlying mechanisms during intrauterine life or throughout the newborn period. The risk for $\mathrm{CP}$ is gestational age dependent, and is much more prevalent among preterm neonates, especially those who are extremely premature born in high and low-income countries.

According to the revised studies, there is a clear difference between well developed and developing countries in potential causes for CP. Despite the fact, there are also similarities for both, such as common risk factors for all countries regardless of socio-economic development, although there are only a few researches available from developing countries. However, overall, the mechanisms for development of PC are still not well defined till now and researches tend to often contradict each other in their results.

Despite some investigational efforts to outline CP situation in Kazakhstan, there is a lot that still needs to be investigated further. Further studies should be planned in order to improve local healthcare system and better manage this truly multifactorial problem.

\section{References}

1. M. Bosanquet, L. Copeland, R. Ware, R. Boyd, Developmental Medicine and Child Neurology, 55 (5), 418-426 (2013)

2. C. Morgan, C. Crowle, T.A. Goyen, C. Hardman, M. Jackman, I. Novak et al., Journal of Paediatrics and Child Health, 52 (1), 54-59 (2016) 
3. H. Smithers-Sheedy, N. Badawi, E. Blair, C. Cans, K. Himmelmann, I. Krägeloh-Mann et al., Developmental Medicine and Child Neurology, 56, 323-328 (2014)

4. M. Stavsky, O. Mor, S. A. Mastrolia, S. Greenbaum, N. G. Than, O. Erez, Frontiers in Pediatrics, 5 (21), 1-10 (2017). DOI: https://doi.org/10.3389/fped.2017.00021

5. P. Singhi, \& A. G. Saini, Journal of tropical pediatrics, 59 (6), 434-440 (2013)

6. M. Gladstone, C. Oliver, \& N. Van den Broek, PLoS One, 10 (3), e0120566 (2015)

7. M. Gladstone, Annals of tropical paediatrics, 30 (3), 181-196 (2010)

8. N. L. Tonkonozhenko, G. V. Klitochenko, P. S. Krivonozhkina, N. V. Maliuzhinskaia, Lekarstvennyi vestnik, 1 (57), 1-8 (2015)

9. A. S. Bangash, M. Z. Hanafi, R. Idrees, \& N. Zehra, JPMA. The Journal of the Pakistan Medical Association, 64 (1), 103-107 (2014)

10. S. Daher, \& L. El-Khairy, East Mediterr Health J, 20 (7), 459-68 (2014)

11. J. Hasegawa, S. Toyokawa, T. Ikenoue, Y. Asano, S. Satoh, T. Ikeda ... \& T. Maeda, PloS one, 11 (1), 1-13 (2016)

12. Australian Cerebral Palsy Register (ACPR) Group, Report of the Australian Cerebral Palsy Register, Birth Years 1993-2006 (ACPR Group, Sydney, 2013)

13. E. Himpens, C. Van den Broeck, A. Oostra, P. Calders, \& P. M. D. P. Vanhaesebrouck, Developmental Medicine \& Child Neurology, 50 (5), 334-340 (2008)

14. S. Y. Yim, C. Y. Yang, J. H. Park, M. Y. Kim, Y. B. Shin, E. Y. Kang, ... \& M. O. Kim, Annals of rehabilitation medicine, 41 (4), 638 (2017)

15. M. Oskoui, F. Coutinho, J. Dykeman, N. Jette, T. \& Pringsheim, Developmental Medicine \& Child Neurology, 55 (6), 509-519 (2013)

16. E. Blair, \& L. Watson, Seminars in Fetal and Neonatal Medicine, 11 (2), 117-125 (2006)

17. A. Colver, C. Fairhurst, P. O. Pharoah, Lancet, 383, 1240-1249 (2014)

18. T. Comans, G. Mihala, L. Sakzewski, R. N. Boyd, \& P. Scuffham, Developmental Medicine \& Child Neurology, 59 (7), 756-761 (2017)

19. E. Shepherd, R. A. Salam, P. Middleton, S. Han, M. Makrides, S. McIntyre, ... \& C. A. Crowther, Cochrane Database of Systematic Reviews, 6, 141 (2018)

20. D. Baud, D. J. Gubler, B. Schaub, M. C. Lanteri, \& D. Musso, The Lancet, 390 (10107), 2099-2109 (2017)

21. A. Ludwig, \& H. Hengel, Eurosurveillance, 14 (9), 19140 (2009)

22. Y. L. Chan, S. Saad, R. Machaalani, B. G. Oliver, B. Vissel, C. Pollock, ... \& H. Chen, Frontiers in molecular neuroscience, 10, 306 (2017)

23. I. Novak, M. Hines, S. Goldsmith, \& R. Barclay, 130 (5), e1285-e1312 (2012)

24. J. H. Ellenberg, \& K. B. Nelson, Developmental Medicine \& Child Neurology, 55 (3), 210-216 (2013)

25. S. McIntyre, D. Taitz, J. Keogh, S. Goldsmith, N. Badawi, \& E. V. E. Blair, Developmental Medicine \& Child Neurology, 55 (6), 499-508 (2013)

26. A. M. Pagnozzi, N. Dowson, J. Doecke, S. Fiori, A. P. Bradley, R. N. Boyd, \& S. Rose, NeuroImage: Clinical, 11, 751-759 (2016)

27. F. Farajdokht, S. Sadigh-Eteghad, R. Dehghani, G. Mohaddes, L. Abedi, R. Bughchechi, ... \& J. Mahmoudi, Brain and cognition, 118, 80-89 (2017)

28. H. G. Taylor, P. A. Filipek, J. Juranek, B. Bangert, N. Minich, \& M. Hack, Developmental neuropsychology, 36 (1), 96-117 (2011)

29. M. S. Durkin, M. J. Maenner, R. E. Benedict, K. Van Naarden Braun, D. Christensen, R. S. Kirby, ... \& M. Yeargin-Allsopp, Developmental Medicine \& Child Neurology, 57 (9), 835-843 (2015)

30. L. Linsell, R. Malouf, J. Morris, J. J. Kurinczuk, \& N. Marlow, Developmental Medicine \& Child Neurology, 58 (6), 554-569 (2016) 
31. S. M. Reid, E. Meehan, S. McIntyre, S. Goldsmith, N. Badawi, D. S. Reddihough, Developmental Medicine and Child Neurology, 58 (2), 25-35 (2016)

32. E. Sellier, M. J. Platt, G. L. Andersen, I. Krägeloh-Mann, J. De La Cruz, C. Cans, ... \& J. Chalmers, Developmental Medicine \& Child Neurology, 58 (1), 85-92 (2016)

33. C.S. Gibson, A. H. MacLennan, W. M. Hague, E. A. Haan, K. Priest, A. Chan et al., American journal of obstetrics and gynecology, 193 (4), 1437 (2005)

34. O. Mor, M. Stavsky, M. Yitshak-Sade, S. A. Mastrolia, R. Beer-Weisel, T. RafaeliYehudai, ... \& O. Erez, American journal of obstetrics and gynecology, 214 (1), 105-e1 (2016)

35. R. Romero, Z. A. Savasan, T. Chaiworapongsa, S. M. Berry, J. P. Kusanovic, S. S. Hassan, ... \& M. Mazor, Journal of perinatal medicine, 40 (1), 19-32 (2012)

36. E. Shepherd, R. A. Salam, P. Middleton, M. Makrides, S. McIntyre, N. Badawi, \& C. A. Crowther, Cochrane Database of Systematic Reviews, 8, 101 (2017)

37. K. B. Nelson, \& E. Blair, New England Journal of Medicine, 373 (10), 946-953 (2015)

38. B. Petterson, K. B. Nelson, L. Watson, \& F. Stanley, Bmj, 307 (6914), 1239-1243 (1993)

39. S. Grunt, L. Mazenauer, S. E. Buerki, E. Boltshauser, A. C. Mori, A. N. Datta, ... \& C. Poloni, Pediatrics, 135 (5), e1220-e1228 (2015)

40. A. H. MacLennan, S. C. Thompson, \& J. Gecz, American journal of obstetrics and gynecology, 213 (6), 779-788 (2015)

41. A. Fattal-Valevski, G. Kenet, M. J. Kupferminc, R. Mesterman, Y. Leitner, E. Rimon, ... \& A. Hassner, Thrombosis research, 116 (2), 133-137 (2005)

42. J. O. Coq, M. Delcour, V. S. Massicotte, O. Baud, \& M. F. Barbe, Developmental Medicine \& Child Neurology, 58, 7-11 (2016)

43. J. Rankin, C. Cans, E. Garne, A. Colver, H. Dolk, P. Uldall et al., Developmental Medicine \& Child Neurology, 52 (4), 345-351 (2010) 